\title{
Visibility-dependent depth of focus for incoherent sinusoidal sources
}

\author{
Ronald J. Pieper, Kannan Raj, and Ting-Chung Poon
}

\begin{abstract}
A visibility-dependent depth of focus is developed for incoherent sinusoidal sources. This analysis is based on a derived relationship between the visibility and the optical transfer function (OTF). The formalism developed is general for any aperture topology and arbitrary orientation of sinusoidal sources. To illustrate the application of the method the cases of an annular aperture and a Gaussian aperture are analyzed. It is found that as the level of defect of defocus increases, the maximum visibility for which a particular spatial frequency can be resolved decreases.
\end{abstract}

\section{Introduction}

The often cited criterion for depth-of-focus is expressed solely in terms of the $f$-number of the lens and the wavelength of the light. Perceived image quality can generally be expected to depend not only on these parameters but also on the spectral content of the object. Assuming that the optical designer knows in advance the highest frequency to be resolved on the image with a minimum allowed visibility, a more precise criterion can be formulated.

The performance of an incoherent imaging system can be specified in terms of its optical transfer function (OTF). Hopkins ${ }^{1}$ and $\mathrm{O}^{\prime} \mathrm{Neill}^{2}$ have previously discussed the frequency response of defocused optical systems and the transfer function of annular apertures, respectively. The OTF approach was used by Kavathekar et al. ${ }^{3}$ to derive the irradiance distribution in the diffraction images of truncated, onedimensional periodic objects with triangular waveform by using incoherent objects and annular diffracting apertures. Tschunko ${ }^{4}$ has also studied the imaging performance of the annular apertures. One significant observation made in this investigation is

When the research was performed, the authors were with the Optical Image Processing Laboratory, the Bradley Department of Electrical Engineering, Virginia Polytechnic Institute and State University, Blacksburg, Virginia 24061. R. J. Pieper is now with the Department of Electrical and Computer Engineering, the Naval Postgraduate School, Monterey, California 93943-5000. K. Raj is with the Optical Computing Laboratory, Department of Electrical and Computer Engineering, George Mason University, Fairfax, Virginia 22030.

Received 4 September 1990

0003-6935/92/070977-10\$05.00/0.

(c) 1992 Optical Society of America. that a higher percentage of the energy is concentrated in the central lobe in the case of annular apertures relative to that observed in the case of circular apertures. Pieper et $a l^{5}{ }^{5}$ have analyzed a resolutiondependent depth of focus for periodic impulses in an incoherent imaging system. In that investigation the Rayleigh criterion was employed as a test for resolution. However, the Rayleigh criterion is not valid when the concept is extended to objects such as sinusoidal inputs. Various response properties of a sine wave input have been examined. ${ }^{6}$ Coltman has examined the out-of-focus imaging properties of sine wave inputs and he concludes that there can be a severe degradation of contrast even at moderate line spacing. Not much emphasis, however, has been placed on using visibility as an important criterion for evaluating the depth of focus.

Here we develop a general formalism for evaluating a visibility-dependent depth of focus with an arbitrary aperture topology and with an arbitrary orientation of sinusoidal sources in an incoherent system. It is shown that the visibility and OTF, evaluated along a particular direction in the Fourier plane, are directly proportional. The deterioration of the highest spatial frequency, with a deviation from focus under conditions of constant visibility, is obtained. Although the analysis is applicable to apertures with noncircular symmetry, here we deal only with Gaussian and annular topologies. The case of Gaussian aperture is shown to be analytically tractable, while the analysis applied to annular apertures is based on an exact formalism ${ }^{7}$ for calculating the OTF for annular apertures under defocused conditions.

In Section II a general description of the concepts motivating the analysis is presented. In Section III the relationship between the OTF and visibility is 
obtained. Two specific cases involving Gaussian and annular aperture transmittances are analyzed and the results are presented in Section IV. Section V presents conclusions. In Appendix A, the relation between the OTF and a line transfer function is derived. In Appendix B, a numerical example illustrating the application of the formalism is presented. In Appendix $\mathrm{C}$ we have prepared, for reader convenience, a partial list of symbols used in this paper.

\section{Background}

\section{A. General Description}

In Fig. 1 the response for two point impulses is shown, and according to the Rayleigh criterion $^{8}$ they can be resolved if

$$
l \geq W
$$

where $W$ is the full width at half-maximum (FWHM) intensity of the incoherent impulse response and $l$ is the center-to-center spacing. Technically the Rayleigh criterion is only defined for impulse-type objects. Nonetheless, a brief examination shows that the Rayleigh criterion cannot be extended to sinusoidal objects. It is apparent from Fig. 2(a) that objects having the sinusoidal variation shown are at the threshold of the Rayleigh criterion. Therefore the criterion will fail if a small amount of dc is introduced, as illustrated by Fig. 2(b). Hence an alternative resolution criterion should be employed.

The coherent impulse response $h_{c}(u, v)$ for an arbitrary aperture $P(x, y)$ (see Ref. 5 ) is given by (see Fig. 3)

$$
h_{c}(u, v)=\left\{h_{d}(x, y) \exp \left[\frac{-j k}{2 F}\left(x^{2}+y^{2}\right)\right] P(x, y)\right\} * h_{d^{\prime}}(x, y),
$$

where $*$ denotes spatial convolution, which is defined as

$$
r * s=\int_{-\infty}^{\infty} \int_{-\infty}^{\infty} s(x, y) r(u-x, v-y) \mathrm{d} x \mathrm{~d} y,
$$

and where the lens shown in Fig. 3 has focal length $F$. The free-space propagation impulse responses are

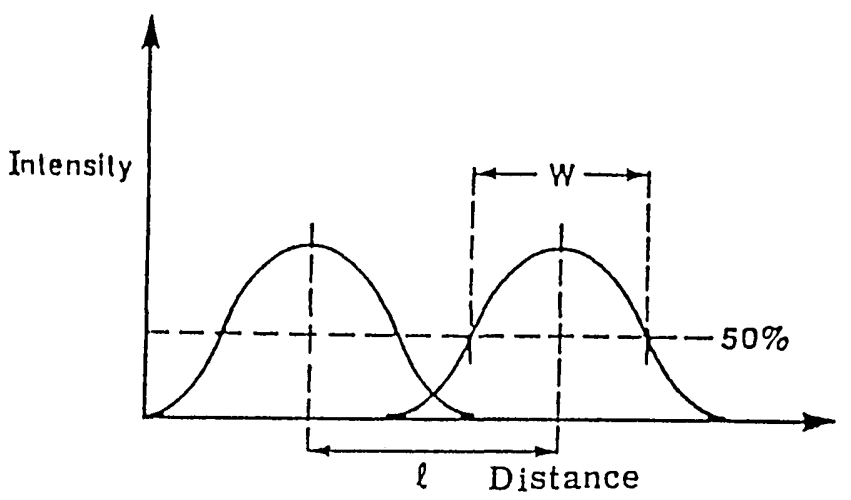

Fig. 1. Two impulse responses with defining parameters for the Rayleigh criterion. given as

$$
\begin{gathered}
h_{d}(x, y)=c_{1} \exp \left[j \frac{k}{2 d}\left(x^{2}+y^{2}\right)\right], \\
h_{d^{\prime}}(x, y)=c_{2} \exp \left[j \frac{k}{2 d^{\prime}}\left(x^{2}+y^{2}\right)\right],
\end{gathered}
$$

where $k=2 \pi / \lambda$ is the propagation constant in free space and $c_{1}$ and $c_{2}$ are complex constants. After defining the out-of-focus defect $\epsilon$ as

$$
\epsilon=\frac{1}{F}-\frac{1}{d}-\frac{1}{d^{\prime}}
$$

and excluding the nonessential complex constants, the coherent impulse response in Eq. (2a) can be explicitly expressed as

$h_{c}(u, v)=\exp \left[j \frac{k}{2 d^{\prime}}\left(u^{2}+v^{2}\right)\right] \mathscr{F}_{x y}\left\{\exp \left[-j \frac{k}{2} \epsilon\left(x^{2}+y^{2}\right)\right] P(x, y)\right\}$,

where the two-dimensional Fourier-transform operator $\mathscr{F}_{x y}$ is defined as

$\mathscr{F}_{x y}[t(x, y)]=\int_{\infty}^{\infty} \int_{\infty}^{\infty} t(x, y) \exp \left[-j 2 \pi\left(f_{x} x+f_{y} y\right)\right] \mathrm{d} x \mathrm{~d} y \equiv T\left(f_{x}, f_{y}\right)$,

and where the spatial frequencies are required to follow the substitution rules $f_{x}=u / \lambda d^{\prime}$ and $f_{y}=$ $v / \lambda d^{\prime}$. The incoherent impulse response is obtained from the magnitude squared of the coherent impulse response $^{9}$

$$
h_{i}(u, v)=\left|h_{c}(u, v)\right|^{2} .
$$

As a result, the complex exponential factor preceding the Fourier transform in Eq. (4) can be dropped. The specification of actual physical parameters can be obviated by introducing a scale transformation in the aperture plane,

$$
\begin{aligned}
& x^{\prime}=x / \mathscr{R}, \\
& y^{\prime}=y / \mathscr{R},
\end{aligned}
$$

a scaled defocus defect

$$
\beta=\mathscr{R} \sqrt{\frac{k \epsilon}{\pi}},
$$

a scale transformation in the image plane [Fig. 4(b)],

$$
\begin{gathered}
\alpha=k u \mathscr{R} / \pi d^{\prime}, \\
\omega=k u \Re / \pi d^{\prime},
\end{gathered}
$$

and finally a normalized aperture transmittance,

$$
p\left(x^{\prime}, y^{\prime}\right)=P\left(\mathscr{R} x^{\prime}, \mathscr{R} y^{\prime}\right) \equiv P(x, y) .
$$




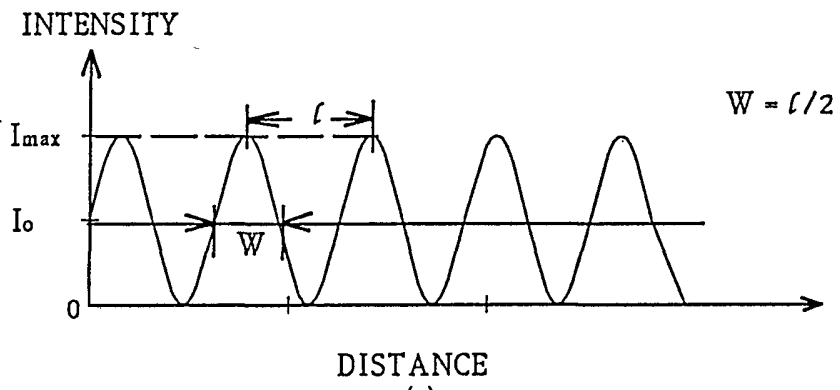

(a)

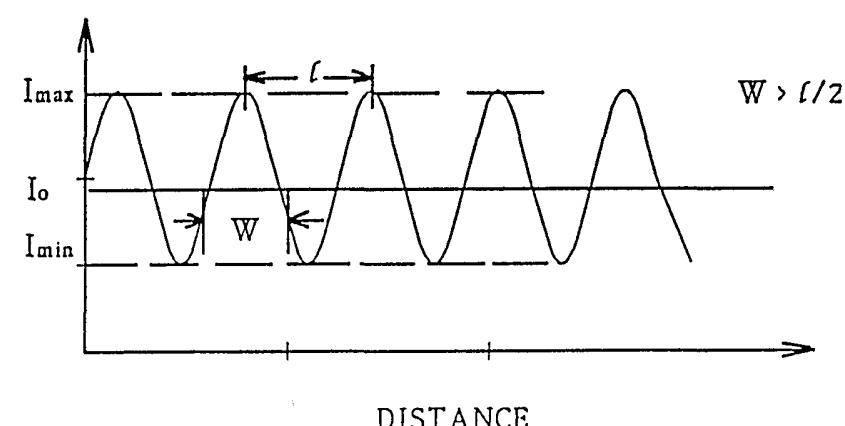

(b)

Fig. 2. (a) Response to sinusoidal input. (b) Rayleigh criterion fails with the addition of a small amount of dc.

In Eqs. (7), $\mathscr{R}$ is an arbitrary scale dimension. As demonstrated in the examples, $\mathscr{R}$ can be specified once an aperture topology is chosen.

After some algebra, ${ }^{5}$ the coherent impulse response Eq. (4) can be written in terms of $\alpha$ and $\omega$, and the result is represented compactly as

$$
\hat{h}_{c}(\alpha, \omega)=\left.\frac{A(P)}{A(p)} \mathscr{F}_{x^{\prime} y^{\prime}}\left\{\exp \left[-j \frac{\pi}{2} \beta^{2}\left(x^{\prime 2}+y^{\prime 2}\right)\right] p\left(x^{\prime}, y^{\prime}\right)\right\}\right|_{f_{x}=\alpha / 2, f_{y}=\omega / 2},
$$

where $A(p)$ is the normalized integrated aperture transmittance, ${ }^{5}$ which is defined as

$$
A(p) \equiv \frac{A(P)}{\mathscr{R}^{2}},
$$

and where the integrated aperture transmittance $A(P)$ is defined as

$$
A(P)=\int_{-\infty}^{\infty} \int_{-\infty}^{\infty} P(x, y) \mathrm{d} x \mathrm{~d} y
$$

It follows from Eqs. (7f) and (8b) that $A(p)$ can also be expressed as

$$
A(p)=\int_{-\infty}^{\infty} \int_{\infty}^{\infty} p\left(x^{\prime}, y^{\prime}\right) \mathrm{d} x^{\prime} \mathrm{d} y^{\prime}
$$

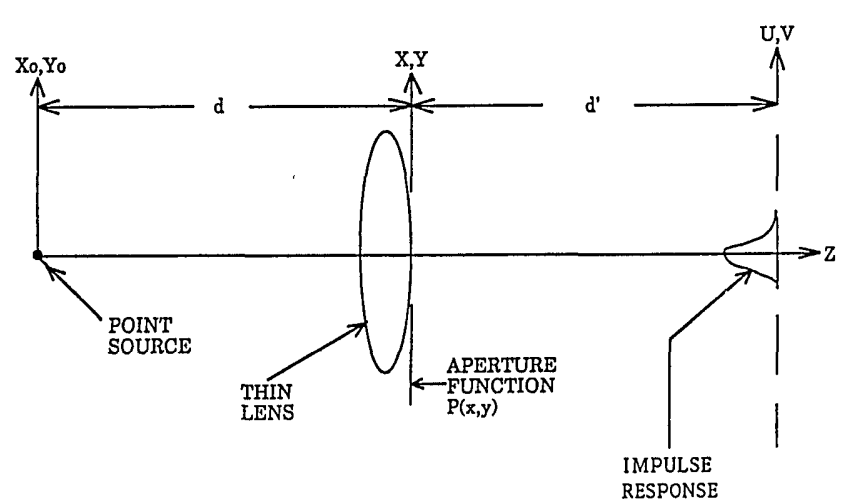

Fig. 3. Optical configuration examined for the determination of the impulse response.
It can be easily demonstrated that for the in-focus conditions, i.e., $\beta=0$, Eq. (8a) satisfies the normalization condition $h_{c}(0,0) / A(P)=1.0$. The ${ }^{\wedge}$ sign in Eq. (8a) over $h_{c}$ and in the rest of the paper indicates that the corresponding functions are expressed in terms of scaled variables $\alpha$ and $\omega$. Below we extend this convention to cover frequency responses written in terms of functions of scaled frequencies. From Eq. (6) the scaled incoherent impulse response is defined as

$$
\hat{h}_{i}(\alpha, \omega)=\left|\hat{h}_{c}(\alpha, \omega)\right|^{2} .
$$

It is worth noting that all the responses and transfer functions discussed herein depend on the deviation from focus. This dependence on $\epsilon$ or equivalently $\beta$, although not explicitly shown, will play an important role in extracting the depth-of-focus information discussed later in the text.

Equation (6) defines the incoherent impulse response. The corresponding expression for the $\mathrm{OTF}^{7}$ is

$$
\mathrm{OTF}=\frac{\mathscr{H}_{i}\left(f_{u}, f_{v}\right)}{\mathscr{H}_{i}(0,0)},
$$

where $\mathscr{L}_{i}$, the incoherent transfer function, ${ }^{9}$ is defined as

$$
\mathscr{H}_{i}\left(f_{u}, f_{v}\right)=\mathscr{F}_{u v}\left[h_{i}(u, v)\right]
$$

where $f_{u}$ and $f_{v}$ are spatial frequencies defined in the $u v$ plane. We use Eqs. (6) and (10b) and the autocorrelation theorem ${ }^{9}$ to express Eq. (10a) as

$$
\operatorname{OTF}\left(f_{u}, f_{v}\right)=\frac{H\left(f_{u}, f_{v}\right) \otimes H\left(f_{u}, f_{v}\right)}{\int_{-\infty}^{\infty}\left|H\left(f_{u}, f_{v}\right)\right|^{2} \mathrm{~d} f_{u} \mathrm{~d} f_{v}}
$$

where $\otimes$ denotes the correlation function as

$$
\begin{gathered}
r \otimes s=\int_{-\infty}^{\infty} \int_{-\infty}^{\infty} r(x, y) s *(x-u, y-v) \mathrm{d} x \mathrm{~d} y \\
H\left(f_{u}, f_{v}\right)=\mathscr{F}_{u v}\left[h_{c}(u, v)\right]
\end{gathered}
$$

is the coherent transfer function. 
The scaled version of the frequency analysis starts from the definition of the scaled coherent transfer function

$$
\hat{H}\left(f_{u}, f_{v}\right) \equiv \mathscr{F}_{\alpha \omega}\left[\hat{h}_{c}(\alpha, \omega)\right] .
$$

After reference to Eqs. (5b), (7d), and (7e) it follows that Eq. (10e) can be expressed as

$$
H\left(f_{\mathrm{a}}, f_{\omega}\right)=\left(\frac{\pi d^{\prime}}{h \mathscr{R}}\right)^{2} \hat{H}\left(f_{\alpha}, f_{\omega}\right),
$$

where

$$
\begin{aligned}
& f_{\alpha}=\frac{\pi d^{\prime}}{k \mathscr{R}} f_{u}, \\
& f_{\omega}=\frac{\pi d^{\prime}}{k \mathscr{R}} f_{v},
\end{aligned}
$$

define the scaled spatial frequencies. The direct substitution of Eq. (11b) into Eq. (10c) leads to the result that

$$
\operatorname{OTF}\left(f_{a}, f_{\omega}\right)=\frac{\hat{\mathscr{Z}} \hat{\mathscr{f}}_{i}\left(f_{\mathrm{a}}, f_{\mathrm{w}}\right)}{\hat{\mathscr{Z}}_{i}(0,0)}
$$

where

$$
\hat{\mathscr{H}}_{i}\left(f_{\alpha}, f_{\omega}\right)=\hat{H}\left(f_{a}, f_{\omega}\right) \otimes \hat{H}\left(f_{\alpha}, f_{\omega}\right) .
$$

In the next section the relationship between the scaled line response and the line transfer function are developed.

\section{B. Scaled Line Response and Line Transfer Function}

Consider an idealized line source making an angle $\theta$ with the $u$ axis and aligned with the $\tilde{u}$ axis, as shown in Fig. 4(a). The line response ${ }^{10}$ is defined as

$$
q^{\theta}(u, v)=\int_{-\infty}^{\infty} \int_{-\infty}^{\infty} h_{i}\left(u-u^{\prime}, v-v^{\prime}\right) \delta\left(v^{\prime} \cos \theta-u^{\prime} \sin \theta\right) \mathrm{d} u^{\prime} \mathrm{d} v^{\prime} .
$$

Although Eq. (15) is expressed in terms of general coordinates $u$ and $v$, it will be shown that it is possible to characterize the line response completely in terms of the variable $\tilde{v}$ measured perpendicular to the source. This is illustrated in Fig. 4(a). The first step is to perform a transformation of variables on Eq. (15). The space coordinates $u$ and $v$ are related to the rotation coordinates $\tilde{u}$ and $\tilde{v}$ [see Fig. 4(a)] as follows:

$$
\begin{aligned}
& u=\tilde{u} \cos \theta-\tilde{v} \sin \theta, \\
& v=\tilde{u} \sin \theta+\tilde{v} \cos \theta .
\end{aligned}
$$

In the text a tilde is used to denote a rotated variable. Substituting Eq. (16) and the corresponding primed equations into Eq. (15) and integrating over $\tilde{v}^{\prime}$ lead to

$$
q^{\theta}(\tilde{v})=\int_{-\infty}^{\infty} h_{i}(\tilde{g} \cos \theta-\tilde{v} \sin \theta, \tilde{g} \sin \theta+\tilde{v} \cos \theta) \mathrm{d} \tilde{g},
$$

where a dummy variable $\tilde{g}$ has been substituted for $\left(\tilde{u}-\tilde{u}^{\prime}\right)$ in Eq. (17). Equation (17) can be written in terms of rotated scaled variables, which are shown in Fig. 4(b). After reference to Eq. (9) and the transformation rules in Eqs. (7d) and (7e), Eq. (17) can be written as

$$
\hat{q}^{\theta}(\tilde{\omega})=\frac{\pi d^{\prime}}{k \mathscr{R}} \int_{-\infty}^{\infty} \hat{h}_{i}(\tilde{\alpha} \cos \theta-\bar{\omega} \sin \theta, \tilde{\alpha} \sin \theta+\bar{\omega} \cos \theta) \mathrm{d} \tilde{\alpha}
$$

where the integration path in Eq. (18) is parallel to the line source shown in Fig. 4(b).

The line transfer function $Q^{\theta}(f)$ associated with the line response is obtained from a one-dimensional

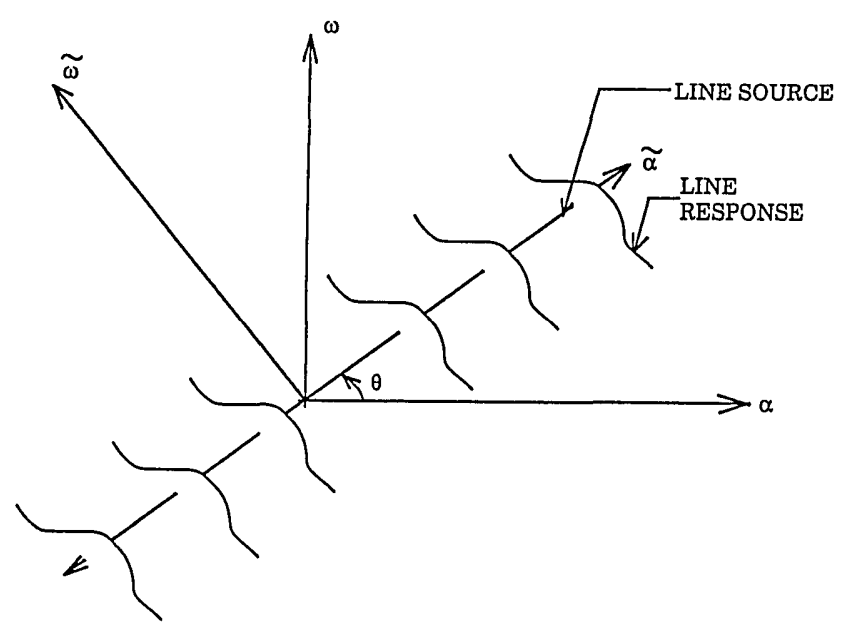

(b)

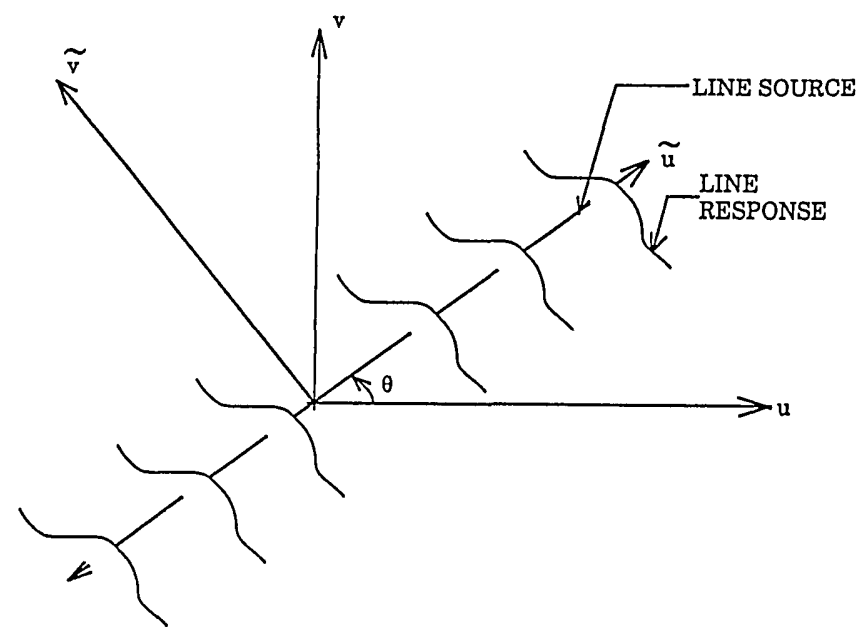

(a)

Fig. 4. (a) Relationship between spatial coordinates $u$ and $v$ and rotated spatial coordinates $\tilde{u}, \tilde{v}$; the line source is oriented along the $\tilde{u}$ axis and at an angle $\theta$ with the $u$ axis. (b) Relationship between scaled spatial coordinates $\alpha$ and $\omega$ and rotated scaled spatial coordinates $\bar{\alpha}$ and $\bar{\omega}$. 
Fourier transform relationship,$^{10}$ which is defined as

$$
Q^{\theta}(f)=\int_{-\infty}^{\infty} q^{\theta}(\tilde{v}) \exp (-j 2 \pi f \tilde{u}) \mathrm{d} \bar{v} .
$$

An equivalent scaled expression for the line transfer function [Eq. (19a)], after reference to Figs. 4(a) and (4b) and Eq. (7d), is given by

$$
\hat{Q}^{0}\left(f_{n}\right)=\left(\frac{\pi d^{\prime}}{k \mathscr{R}}\right) \int_{-\infty}^{\infty} \hat{q}^{\theta}(\tilde{\omega}) \exp \left(-j 2 \pi f_{n} \tilde{\omega}\right) \mathrm{d} \tilde{\omega},
$$

or, more compactly, is represented as

$$
\hat{Q}^{\ominus}\left(f_{n}\right)=\left(\frac{\pi d^{\prime}}{k \mathscr{R}}\right) \mathscr{F}_{\tilde{\omega}}\left[\hat{q}^{\theta}(\tilde{\omega})\right\},
$$

where the scaled frequency $f_{n}$ is given by

$$
f_{n}=\frac{\pi d^{\prime}}{k \mathscr{R}} f \text {. }
$$

A comparison of Eq. (12) with Eq. (19d) shows that $f_{n}$ is equivalent to the scaled spatial frequency associated with the $\tilde{\omega}$ axis shown in Fig. 4(b). In order to illustrate the physical significance of Eq. (19d), we consider, as an example, a circular aperture system characterized by a radius $R$. If we take $\mathscr{R}=R$ then

$$
\frac{k R}{\pi d^{\prime}}=\frac{2 R}{\lambda d^{\prime}}=2 f_{c}
$$

where $f_{c}$ is the conventional frequency normalization factor used in the analysis of the frequency response of an optical system with circular apertures. ${ }^{11}$ For circular aperture systems described in terms of frequencies normalized by $f_{c}$, the peak normalized frequency with nonzero values of the OTF is two. It follows from Eqs. (19d) and (20) that with the normalization factor employed herein $\left(2 f_{c}\right)$, the peak value of $f_{n}$ generating nonzero values of the circular aperture's OTF will be 1 . Section III describes a resolution criterion that is adopted for sinusoidal sources.

\section{Criterion for Resolution for Sinusoidal Sources}

In Section II it was shown that the Rayleigh criterion would not apply to sinusoidal sources. Therefore an alternate image evaluation criterion that can guarantee satisfactory resolution is needed. The resolution criterion to be applied depends on the minimum contrast that can be perceived under defocused conditions. Contrast is usually quantified in terms of the visibility $V$, which is defined as ${ }^{11}$

$$
V=\frac{I_{\max }-I_{\min }}{I_{\max }+I_{\min }}
$$

where $I_{\max }$ and $I_{\min }$ are the maximum and minimum intensities within an image. Definition (21) can be applied to either source or image intensities. It is confirmed by inspection of Eq. (21) that, in the image plane, geometrical magnification will not affect visibil- ity. As a consequence of the above observation the visibility of the ideal geometrical image, taken as our input, will be compared with the visibility of the diffraction-limited output image.

For an input having an intensity with a sinusoidal variation along the $v$ axis [Fig. 4(a)], the input intensity can be defined as

$$
I_{\text {in }}=I_{o}\left[1+m \cos \left(2 \pi f_{o} v\right)\right],
$$

where $I_{o}$ is the average input intensity, $m$ is the input signal visibility, and $f_{o}$ is the input spatial frequency of the sinusoidal variation. In Appendix A it is shown that if the only nontrivial spatial frequency is $f_{v}$ then, according to Eq. (A4), the correct line response angle is zero. Since the variation of the input signal is along the $v$ axis it is necessary to use $\theta=0$ to obtain the correct line transfer function. In order to lighten the notation the superscript $\theta$ is dropped in the case $\theta=0$. For an input defined by Eq. (22), the output is given as

$$
I_{\text {out }}=I_{o}\left(Q(0)+m\left|Q\left(f_{o}\right)\right| \cos \left\{2 \pi f_{o} v+\operatorname{Arg}\left[Q\left(f_{o}\right)\right]\right\}\right),
$$

where

$$
Q\left(f_{o}\right)=\left|Q\left(f_{o}\right)\right| \exp \left\{-j \operatorname{Arg}\left[Q\left(f_{o}\right)\right]\right\},
$$

and $\operatorname{Arg}[\cdot]$ stands for the argument of. From Eq. (23) the maximum and minimum output intensities are given by

$$
\begin{aligned}
& I_{\max }=|Q(0)|+m\left|Q\left(f_{o}\right)\right|, \\
& I_{\min }=|Q(0)|-m\left|Q\left(f_{o}\right)\right|,
\end{aligned}
$$

and therefore, from Eqs. (21), (25a), and (25b), the output visibility is given as

$$
V=m \frac{\left|Q\left(f_{o}\right)\right|}{|Q(0)|}
$$

These steps can be generalized easily for any arbitrary angle $\theta$ by repeating the analysis in terms of $\tilde{u}$ and $\tilde{v}$, starting from the generalized form of Eq. (23) [i.e., $v \rightarrow \tilde{v}$ and $Q\left(f_{o}\right) \rightarrow Q^{\theta}\left(f_{o}\right)$ ]. This leads to

$$
V=m \frac{\left|Q^{\theta}(f)\right|}{\left|Q^{\theta}(0)\right|}
$$

The corresponding version, which is written in terms of the normalized frequency, that follows from Eq. (19) is

$$
V=m \frac{\left|\hat{Q}^{\theta}\left(f_{n o}\right)\right|}{\left|\hat{Q}^{\theta}(0)\right|}
$$

where $\hat{Q}^{\theta}$ is defined by Eq. (19b). This shows explicitly the relation of the output visibility to the line transfer function for sinusoidal inputs. As shown in Appendix $\mathrm{A}$, the line transfer function is directly proportional to 
the OTF, which is evaluated along a specific direction in the Fourier plane. After combining the result of Eq. (A6) with Eq. (26c) it follows that

$$
V=m_{\text {OTF }}\left(-f_{n} \cos \theta, f_{n} \sin \theta\right) .
$$

Therefore the dependence of the visibility on the frequency of the input signal can be determined from any of the forms provided in Eqs. (26).

In the next section we show how the visibility is related to the spatial frequencies and defocus parameter $\beta$ in the case of Gaussian and annular apertures. The development of these two cases reflect somewhat different, but equivalent, approaches leading to depthof-focus curves. In the Gaussian aperture case, the visibility is obtained from Eq. (26c). In the annular aperture case, direct use is made of the visibility's relation to the OTF [Eq. (26d)]. As demonstrated by all forms of Eqs. (26) the numerical results generated after assuming $m=1.0$ can be applied to any arbitrary input visibility $m<1.0$ through the visibility scaling rule $V \rightarrow V / m$.

\section{Specific Aperture Topologies}

In this section we consider the pupil functions that are radially symmetric. For each case of pupil function considered, a relationship between the scaled spatial frequency $f_{n}$, the defocus defect $\beta$, and the visibility $\nu$ is obtained.

\section{A. Gaussian Aperture}

The Gaussian aperture transmittance profile [see Fig. $5(\mathrm{a})]$ is represented as

$$
P(x, y)=\exp \left(\frac{-r^{2}}{R^{2}}\right) .
$$

If we take the normalizing dimension to be $\mathscr{R}=R$, the scaled function is expressed as

$$
p\left(x^{\prime}, y^{\prime}\right)=\exp \left(-r^{\prime 2}\right) .
$$

We also note that

$$
A(p)=2 \pi \int_{0}^{\infty} \exp \left(-r^{\prime 2}\right) r^{\prime} \mathrm{d} r^{\prime}=\pi .
$$

From Eqs. (8) and (9) or Ref. 5, the incoherent impulse response is found to be

$$
\hat{h}_{i}(\alpha, \omega)=\frac{\pi^{2} \mathscr{R}^{4}}{\left[1+\left(\frac{\pi}{2} \beta^{2}\right)^{2}\right]} \exp \left[-\frac{2 \pi^{2}\left(\alpha^{2}+\omega^{2}\right)}{F(\beta)}\right],
$$

where $F(\beta)$ is given by

$$
F(\beta)=4+\left(\pi \beta^{2}\right)^{2} .
$$

Because of the circular symmetry in the impulse response [Eq. (30)], the line response [Eq. (17b)] can be evaluated along any direction in the $\alpha-\omega$ plane and the result has to be independent of the particular direction chosen. For convenience we choose the line in Fig. 4(b) that corresponds to $\theta=0$ in Eq. (18). The line response then becomes

$$
\hat{q}(\omega)=2 \sqrt{2 \pi^{5}} \frac{d^{\prime} \mathscr{R}^{3}}{k \sqrt{F}(\beta)} \exp \left[-\frac{2 \pi^{2} \omega^{2}}{F(\beta)}\right] .
$$

The corresponding line transfer function, from Eqs. $(19 \mathrm{~b})$ and (32), is therefore

$$
\hat{Q}\left(f_{n}\right)=\frac{2 \pi^{3} d^{\prime} \mathscr{R}^{3}}{k^{2}} \exp \left[-f_{n}^{2} \frac{F(\beta)}{2}\right] .
$$

From the above, the visibility is obtained, according to Eq. (26c), as

$$
V=m\left|\frac{2 \hat{Q}\left(f_{n}\right)}{\hat{Q}(0)}\right|=m \exp \left[-f_{n}^{2} \frac{F(\beta)}{2}\right] .
$$

We solve Eq. (34) for $f_{n}$ and obtain

$$
f_{n}=\left\{\left.\frac{\ln \frac{m}{V}}{2\left[1+\left(\frac{\pi}{2} \beta^{2}\right)^{2}\right]}\right|^{1 / 2} .\right.
$$

This is a closed-form expression that directly relates the output visibility, the input visibility, and the defocus parameter to the normalized frequency. The normalized frequency is plotted as a function of the defocus parameter $\beta$ for various visibility ratios $V / m$ (see Fig. 6). In our calculations the input visibility $m$ is taken as unity. The following interpretation can be

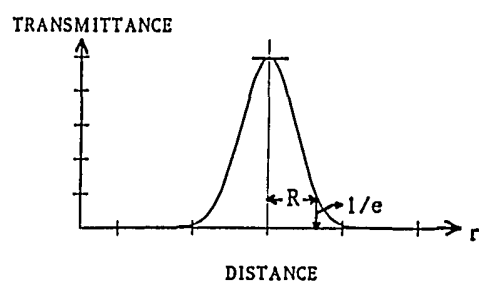

(a)

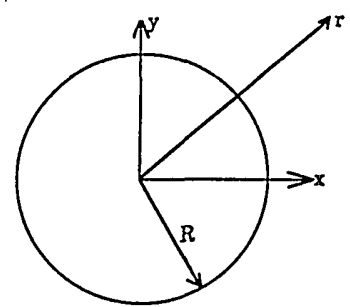

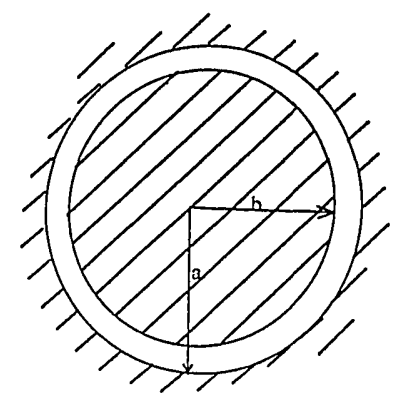

(b)

Fig. 5. (a) Gaussian aperture. (b) Annular aperture. 


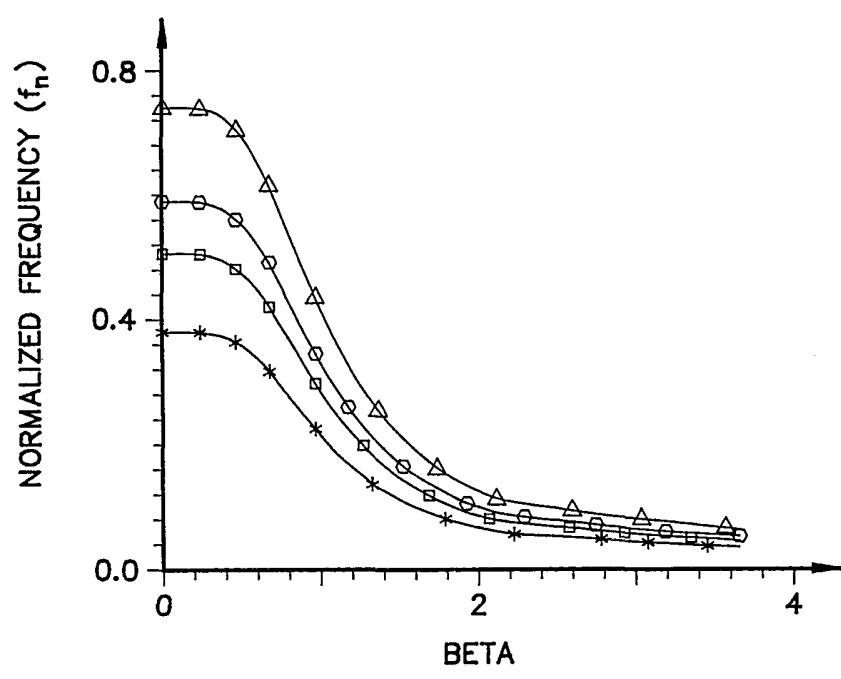

$$
\begin{aligned}
& \triangle \triangle \triangle V=0.25 \quad \rightarrow-0 V=0.33 \\
& \rightarrow \square-V=0.50 \quad-* * * V=0.75
\end{aligned}
$$

Fig. 6. Depth-of-focus curves for Gaussian aperture.

made from the curves. The maximum spatial frequency that meets the criterion of fixed output visibility decreases as the optical system moves away from focus.

In Appendix B a numerical example, using Fig. 6, is presented to illustrate the use of such curves in calculating the depth of focus.

\section{B. Annular Aperture}

A generalized pupil function for circular apertures, which incorporates the defect of defocus, is defined as $^{7}$

$$
\mathscr{P}_{s}=P_{\curvearrowright}\left(f_{\alpha}, f_{\omega}\right) \exp \left[-j 2 \pi \beta^{2}\left(f_{\alpha}^{2}+f_{\omega}^{2}\right)\right],
$$

where

$$
\begin{gathered}
P_{s x}\left(f_{\alpha}, f_{\omega}\right)=\operatorname{circ}\left[\frac{\left(f_{\alpha}^{2}+f_{\omega}^{2}\right)^{1 / 2}}{2 \hat{a}}\right] ; \\
\hat{a}=\frac{a}{\mathscr{R}}
\end{gathered}
$$

is the circular aperture radius that is scaled by the factor $\mathscr{R}$. These equations are obtained from those in Ref. 7 after letting $(r, q) \rightarrow\left(2 f_{\alpha}, 2 f_{\omega}\right)$. For annular apertures [see Fig. 5(b)], the generalized pupil function is defined as

$$
\mathscr{P}=\mathscr{P}_{\mathscr{P}}-\mathscr{P}_{\mathscr{P}},
$$

where the mathematical form for $P_{\mathscr{B}}$ is that given in Eqs. (36) after letting $a \rightarrow b$. By applying the autocorrelation operation and using Eq. (14) in Eq. (37), we obtain, after normalization, the $\mathrm{OTF}^{7}$ :

$$
\text { OTF }=\frac{1}{\pi\left(a^{2}-b^{2}\right)}\left[\mathscr{P}_{\mathscr{A}} \otimes \mathscr{P}_{\mathscr{S}}+\mathscr{P}_{\mathscr{B}} \otimes \mathscr{P}_{\mathscr{B}}-2 \operatorname{Re}\left(\mathscr{P}_{\mathscr{B}} \otimes \mathscr{P}_{\mathscr{A}}\right)\right]
$$

where $\operatorname{Re}(\cdot)$ stands for the real part of. In the calculation of Eq. (38) it is appropriate to take $\mathscr{R}=a$, as in Eq. (36c), so that, as discussed at the end of Section II, the maximum spatial frequency for nonzero OTF becomes unity.

As seen in Section III, the visibility is related to the OTF. For annular apertures with different obscuration ratios (the obscuration ratio is defined as the ratio of the inner radius to the outer radius, i.e., $b / a$ ), an exact relationship for the OTF has been derived. The approach adopted there was to evaluate the cross correlation, without approximation, of two different circular apertures in the context of a two-pupil interactive system. ${ }^{12} \mathrm{~A}$ block of data that numerically defines the OTF for various defocus parameters as a function of spatial frequency was generated by Eq. (38). For an example, see Fig. 7. The defocus parameter $W$ is related to the defocus defect $\beta$ as $W=\pi \beta^{2}$. Results given in Ref. 7 pertaining to an obscuration ratio of 0.5 are reproduced in Fig. 7 for reference. Note that the results in Ref. 7 follow the more conventional frequency normalization scheme and therefore are expressed in terms of spatial frequencies which are normalized to a maximum value of two. As previously explained, for the examples presented in this investigation the normalized maximum spatial frequency is taken to be unity.

Since the OTF is related to the visibility, once a particular visibility value has been chosen the corresponding normalized spatial frequency can be obtained by a linear interpolation of the spatial frequency versus the OTF curve. In this way a new set of

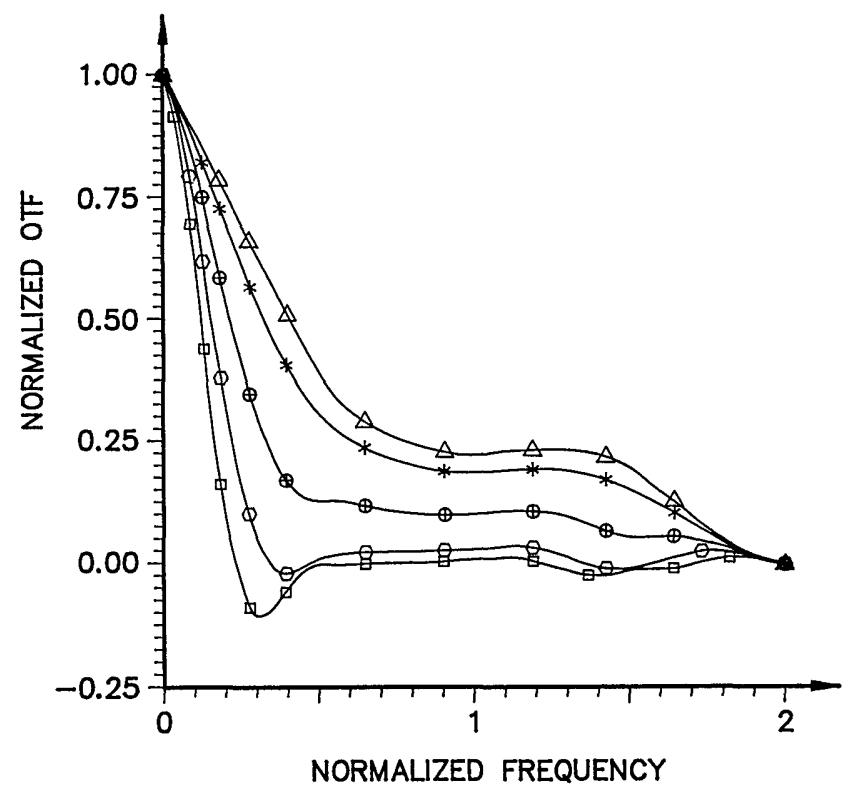

$$
\begin{gathered}
\triangle \triangle \triangle W=0.00 \rightarrow W=12.0 \rightarrow W=16.0 \\
* * * W=4.00 \rightarrow W=8.00 \\
\text { OBSCURATION RATIO }=0.5
\end{gathered}
$$

Fig. 7. OTF curve for annular aperture; obscuration ratio 0.5 (after Ref. 7). 
curves that relates visibility to the spatial frequency for various defocus values can be generated. Three plots corresponding to an input visibility of one (i.e., $m=1$ ) and obscuration ratios of 0.0 [Fig. 8(a)], 0.5 [Fig. 8(b)], and 0.9 [Fig. 8(c)], respectively, have been generated. The conclusions drawn from these curves are similar to those of the Gaussian case. A particular spatial frequency can be resolved at higher visibility only under smaller defocus conditions. Also, it is seen from Fig. 8(c) that as the obscuration ratio increases the depth of focus increases. ${ }^{13}$ This is indicated by the flat region in the figure. However, its apparent that
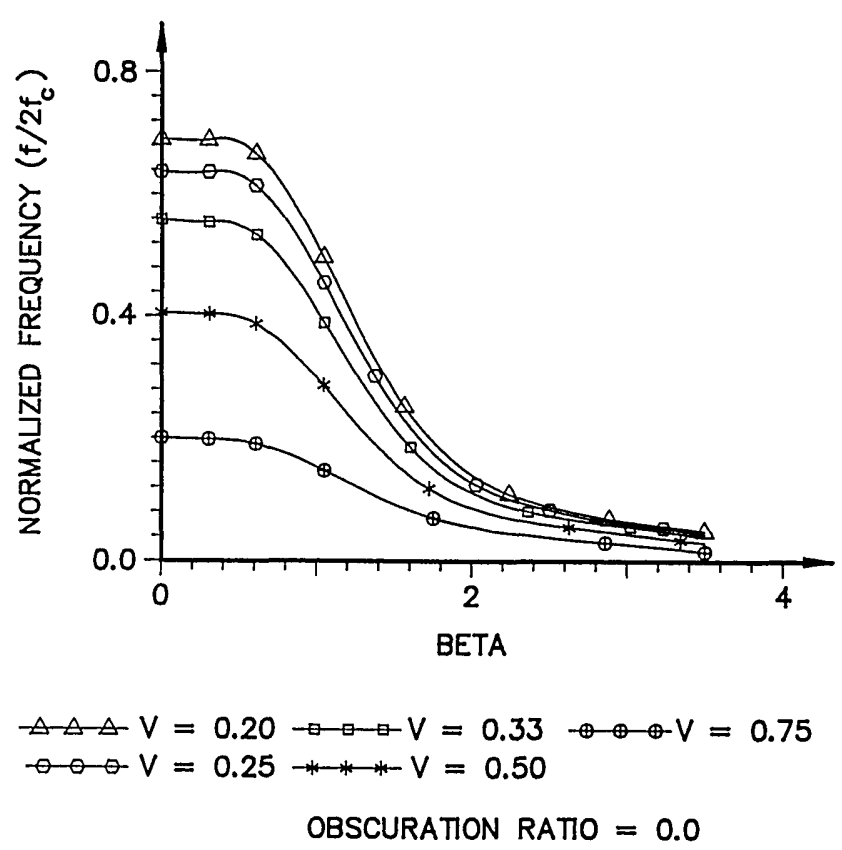

(a)

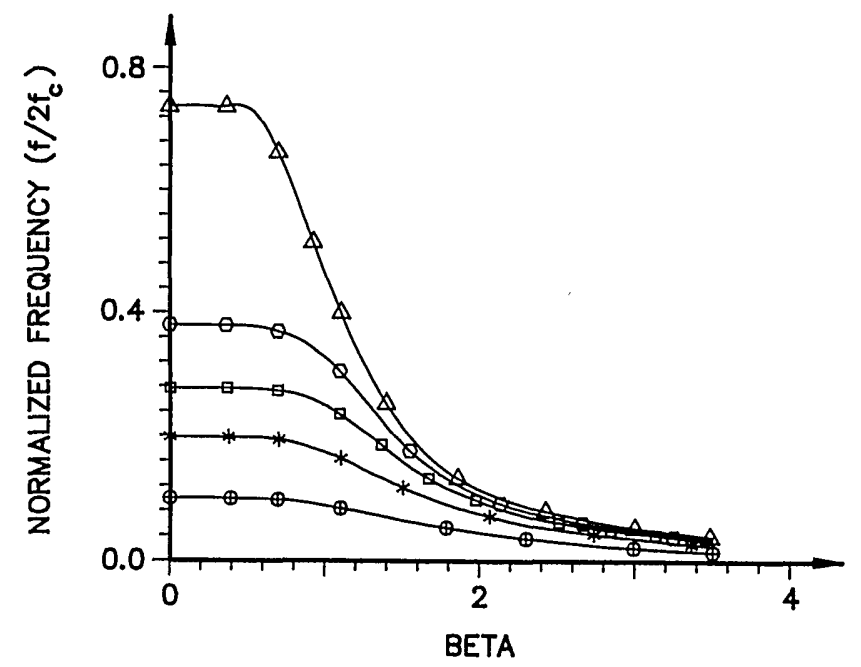

$$
\begin{gathered}
\triangle \triangle V=0.20 \rightarrow-V=0.33 \leftrightarrow-V=0.75 \\
\square \vee V=0.25 \rightarrow-V=0.50 \\
\text { OBSCURATION RATIO }=0.5
\end{gathered}
$$

(b) much lower spatial frequencies are represented in Fig. 8(c) with respect to those shown in Figs. 8(a) and $8(\mathrm{~b})$. This is a direct consequence of the relationship between the output visibility and the associated normalized frequency. Under conditions of fixed normalized frequency the visibility would deteriorate as the obscuration factor of the annulus increases. If the visibility values are fixed, as in the comparison of Figs. 8(a), 8(b), and 8(c), then the associated normalized frequency will decrease with an increase in the obscuration factor. Although the results ${ }^{5}$ for annular apertures, which are based on the Rayleigh criterion

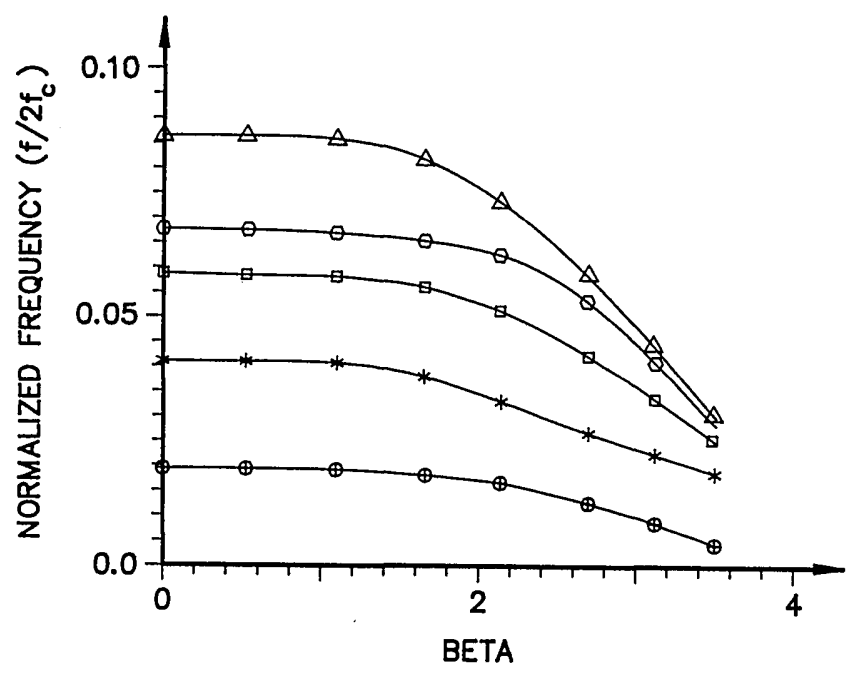

$\triangle \triangle \triangle V=0.20 \rightarrow-V=0.33 \rightarrow-Q-V=0.75$
$\square \triangle V=0.25 \rightarrow 0.50$
$\begin{gathered}\text { OBSCURATION RATIO }=0.9 \\ \text { (c) }\end{gathered}$
Fig. 8. (a) Depth-of-focus curves for the annular aperture with obscuration ratios of (a) 0.0 ; $\quad$ (b) 0.5 ; (c) 0.9 . 
for impulse type sources, also demonstrate an extended depth-of-focus feature, none of the effects associated with deterioration in contrast is evident.

\section{Conclusions}

Here we have outlined a visibility-based criterion for evaluating the depth of focus for sinusoidal sources. Furthermore, it has been found that visibility is directly proportional to the defocused OTF that is evaluated along a specific direction in the spatial frequency plane. In the case of Gaussian and annular pupil functions, the spatial frequency is plotted as a function of the defocus defect parameter $\beta$ for various visibility values. The case involving Gaussian pupils is shown to be analytically tractable, while that dealing with an annular pupil is analyzed by using an exact formalism ${ }^{7}$ for evaluating the OTF. It has been found that as the amount of defocus increases the maximum spatial frequency that meets the visibility requirement is lowered.

Several extensions of this research are currently under investigation. First, the analysis presented here can be extended to arbitrary periodic inputs, since any input can be expressed as a weighted sum of its sinusoidal components. Second, as mentioned in Section I, the Rayleigh criterion has also been applied to produce depth-of-focus curves. ${ }^{5}$ For the examples taken, the results that are based on the visibility criterion provided a more conservative estimate of the depth of focus. One possible extension of this work would be to perform a depth-of-focus comparison on objects for which both criteria apply.

\section{Appendix A}

In this appendix we derive, in terms of unscaled variables, a relationship between the OTF and the line transfer function. It is also shown that this result can be extended to the scaled variable OTF that is introduced in the formalism. From Eqs. (17) and (19) we find that the frequency response can be expressed as

$$
\begin{aligned}
Q^{\theta}(f)=\int_{-\infty}^{\infty} \int_{-\infty}^{\infty} h_{i}(\tilde{g} \cos \theta-\tilde{v} \sin \theta, \tilde{g} \sin \theta+\tilde{v} \cos \theta) \\
\times \exp [-j 2 \pi f(\tilde{v} \cos \theta-\tilde{g} \sin \theta)] \mathrm{d} \tilde{v} \mathrm{~d} \tilde{g} .
\end{aligned}
$$

After allowing $\tilde{g} \rightarrow \tilde{u}$ in Eq. (16), Eq. (A1) can be rewritten as

$$
Q^{\theta}(f)=\int_{-\infty}^{\infty} \int_{-\infty}^{\infty} h_{i}(u, v) \times \exp [-j 2 \pi f(v \cos \theta-u \sin \theta)] \mathrm{d} u \mathrm{~d} v .
$$

Therefore, according to definition (10b),

$$
Q^{\theta}(f)=\mathscr{H}_{i}\left(f_{u}, f_{v}\right),
$$

where the spatial frequencies must satisfy the conditions

$$
\begin{gathered}
f_{u}=-f \sin \theta, \\
f_{v}=f \cos \theta .
\end{gathered}
$$

Equation (A3) provides a means of calculating the line transfer function for an arbitrary angle of line source. With reference to Fig. 4(a) and Eq. (A4), we conclude that for a line source making an angle $\theta$ with the $u$ axis, Eq. (A3) specifies the frequency response along an axis normal to the line source. This observation is consistent with the dependence of the line response [Eq. (17)] on the coordinate measured along the axis that is normal to the line source. By using Eq. (A3), Eq. (10a) becomes

$$
\mathrm{OTF}(-f \sin \theta, f \cos \theta)=\frac{Q^{\theta}(f)}{Q^{\theta}(0)} .
$$

Equation (A5) thus relates the OTF with the line transfer function for any arbitrary angle $\theta$. In terms of $f_{n}$ [Eq. (19d)], the scaled OTF can be written as

$$
\widehat{\mathrm{OTF}}\left(-f_{n} \sin \theta, f_{n} \cos \theta\right)=\frac{\hat{Q}^{\theta}\left(f_{n}\right)}{\hat{Q}^{\theta}(0)},
$$

where $Q^{\theta}\left(f_{n}\right)$ is evaluated from Eq. (19b).

\section{Appendix B}

In this appendix an application of the depth-of-focus curves is presented. The curves pertaining to the Gaussian aperture transmittance (Fig. 6) are used as reference. The relevant parameters that are chosen are as follows:

$$
\begin{array}{ll}
F=10 \mathrm{~cm}, & d=20 \mathrm{~cm}, \\
\lambda=0.6 \times 10^{-4} \mathrm{~cm}, & \mathscr{R}=R=4.0 \mathrm{~cm} .
\end{array}
$$

From Eq. (20a), we have

$$
f_{n}=\frac{\lambda d^{\prime}}{2 R} f
$$

Now let us assume that the depth of focus is small enough so that $d^{\prime} \approx d_{F^{\prime}}$ (where $d_{F^{\prime}}$ satisfies the in-focus condition $\left.1 / d+1 / d_{F^{\prime}}=1 / F\right)$.

By evaluating any curve in Fig. 6 at the value of the normalized spatial frequency indicated by Eq. (B1), we can find the corresponding defect parameter $\beta$ for the associated visibility. The defocus defect $\epsilon$ can then be related, with Eq. (7c), to the defocus defect parameter $\beta$ :

$$
\epsilon=\left(\frac{\beta}{R}\right)^{2} \frac{\lambda}{2} .
$$

Also it is seen from Eq. (3c) that the out-of-focus defect can be written as

$$
\epsilon=\frac{d_{F^{\prime}}-d^{\prime}}{d_{F^{\prime}} d^{\prime}} \equiv \frac{\Delta d^{\prime}}{d_{F^{\prime}} d^{\prime}} \approx \frac{\Delta d^{\prime}}{d_{F^{\prime}}{ }^{2}} .
$$

The combination of Eqs. (B2) and (B3) leads to an 
Table I. Maximum Deviation $\Delta d^{\prime}$ for Various Visibilities

\begin{tabular}{cccc}
\hline $\begin{array}{c}\text { Spatial } \\
\text { Frequency } \\
(\times 1000\end{array}$ & $\begin{array}{c}\text { Visibility } \\
\text { Defocus }\end{array}$ & $\begin{array}{c}\text { Maximum } \\
\text { Pefocus }\end{array}$ \\
cycles $/ \mathrm{mm})$ & $V$ & $\beta$ & $\Delta d^{\prime}$ (in $\left.\lambda\right)$ \\
\hline 0.15 & 0.75 & 1.24 & 19.22 \\
& 0.50 & 1.45 & 26.28 \\
& 0.33 & 1.60 & 32.00 \\
0.30 & 0.25 & 1.79 & 40.05 \\
& 0.75 & 0.74 & 6.85 \\
& 0.50 & 0.95 & 11.28 \\
& 0.33 & 1.10 & 15.13 \\
& 0.25 & 1.23 & 18.91 \\
\hline
\end{tabular}

expression for the maximum deviation from focus:

$$
\Delta d^{\prime}=\left(\frac{\beta d_{F^{\prime}}}{R}\right)^{2} \frac{\lambda}{2}
$$

From the relation shown in Eq. (B4), and from $\beta$ as taken from Fig. 6, the maximum deviation from focus that one can tolerate can be calculated. In Table I the maximum deviation $\Delta d^{\prime}$ that is associated with the spatial frequencies of 150 and 300 cycles $/ \mathrm{mm}$, respectively, are listed for various visibilities. From Table I we see that the depth of focus is of the order of a few wavelengths. This result validates the assumption made at the beginning that $d^{\prime} \approx d_{F^{\prime}}$. Furthermore, it is apparent from the table that as the minimum visibility increases the maximum deviation from focus decreases.

The classical depth-of-focus expression depends only on the $f$-number, i.e., $F /(2 R)$, and the wavelength. ${ }^{11}$ Under the approximate condition $d_{F}{ }^{\prime} \approx F$ expression (B4) exhibits, for a fixed normalized defocus defect, the correct dependencies on both the $f$-number and the wavelength.

\section{Appendix C: List of Symbols}

$q$ Line response

$\stackrel{\text { Line transfer function }}{ }$

$u, v$ Spatial coordinates in the two-dimensional image plane

$\alpha, \omega$ Scaled spatial coordinates in the two-dimensional image plane

$f$ Spatial frequency for the line transfer function

$f_{\alpha}, f_{\omega}$ Scaled spatial frequencies in the two-dimensional image plane $\epsilon, \beta$ Out-of-focus parameters

$\mathscr{F}$ Fourier-transform operator

$h_{c}$ Coherent impulse response

$h_{i}$ Incoherent impulse response

$H$ Coherent transfer function

$\mathscr{H}_{i}$ Incoherent transfer function

$v$ Output visibility

$m$ Input visibility

* Convolution operation

$\otimes$ Correlation operation

- Denotes a rotated coordinate

- Denotes dependence on scaled coordinates

This research was supported in part under NSF grant No. ECS-8813115 and in part by the Fiber and Electro-Optics Research Center at Virginia Tech.

\section{References}

1. H. H. Hopkins, "Frequency response of a defocused optical system," Proc. R. Soc. London Ser. A 231, 91-103 (1955).

2. E. L. O'Neill, "Transfer function of an annular aperture," J. Opt. Soc. Am. 46, 285-288 (1956).

3. A. K. Kavathekar, G. S. Bhatnagar, and K. Singh, "Diffraction images of truncated periodic objects formed by a diffractionlimited imaging system with an annular aperture," Indian J. Pure Appl. Phys. 12, 138-142 (1974).

4. H. F. A. Tschunko, "Imaging performance of annular apertures," Appl. Opt. 13, 1820-1823 (1974).

5. R. J. Pieper, J. Park, and T.-C. Poon, "Resolution-dependent depth of focus for an incoherent imaging system," Appl. Opt. 27, 2040-2047 (1988).

6. J. W. Coltman, "The specification of imaging properties by response to a sine wave input," J. Opt. Soc. Am. 44, 468-471 (1954).

7. R. J. Pieper and T.-C. Poon, "Optical transfer functions for defocused two-pupil systems involving annular geometries," J. Mod. Opt. 37, 2055-2072 (1990).

8. E. Hecht and A. Zajac, Optics (Addison-Wesley, Reading, Mass., 1976), Chap. 9, pp. 309-310.

9. J. W. Goodman, Introduction to Fourier Optics (McGraw-Hill, New York, 1968), Chap. 6, pp. 112-119.

10. A. Papoulis, Systems and Transforms with Applications in Optics (Krieger Publishing, Melbourne, Fla., 1968), Chap. 3, pp. 55-56.

11. M. Born and E. Wolf, Principles of Optics (McGraw-Hill, New York, 1968), Chap. 7, p. 267.

12. T.-C. Poon, "Scanning holography and two-dimensional image processing by acousto-optic two-pupil synthesis," J. Opt. Soc. Am. A 2, 521-526 (1985).

13. T.-C. Poon and M. Motamedi, "Optical/digital incoherent image processing for extended depth of field," Appl. Opt. 26, $4612-4615$ (1987). 